\title{
Analytical results for hadronic contributions to the muon $g-2$
}

\author{
Johan Bijnens* \\ Lund University \\ E-mail: bijnens@thep.lu.se \\ Nils Hermansson-Truedsson ${ }^{\dagger}$
Lund University
E-mail: nils.hermansson-truedssonethep. lu.se
}

\section{Antonio Rodríguez-Sánchez}

Lund University

E-mail: antonio.rodriguez@thep.lu.se

This talk discusses two analytical calculations relevant for the Standard Model calculation of the muon $g-2$. The first part is the recent derivation of the quark-loop as the first term in a well-defined operator-product expansion for the short-distance part of the hadronic light-by-light contribution, as well as the calculation of the next term. The second part is the calculation of finite volume effects relevant for lattice QCD calculations of the electromagnetic contribution to the lowest-order hadronic vacuum-polarization contribution and the proof they only start at $1 / L^{3}$.

European Physical Society Conference on High Energy Physics - EPS-HEP2019 -

10-17 July, 2019

Ghent, Belgium

\footnotetext{
* Speaker.

${ }^{\dagger}$ Present address: Universität Bern, E-mail: nils@itp.unibe.ch
} 


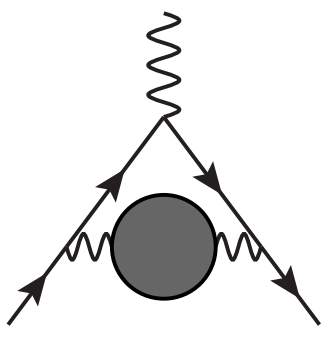

(a) LO-HVP

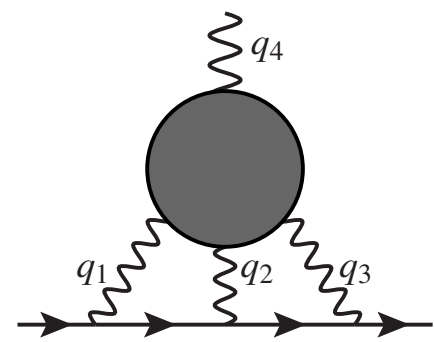

(b) HLbL

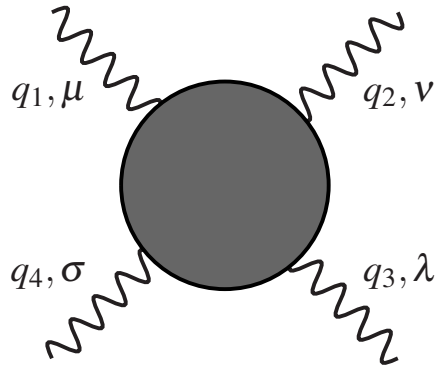

(c) $\Pi^{\mu \nu \lambda \sigma}$

Figure 1: Hadronic contributions to the muon $g-2$.(a) HVP, (b) HLbL, (c) The vector four-point function

\section{Introduction}

The muon anomalous magnetic moment is among the most precisely measured quantities in particle physics. The final result [1] differs from the Standard Model prediction by 3.5-4 $\sigma$, see e.g. [2]. An ongoing experiment at FNAL aims to increase the measurement precision by about a factor of four and there is an experiment under development with an innovative approach at J-PARC.

It is thus imperative that the theoretical error should be brought down to the same level of precision. The theoretical error is dominated by the hadronic contributions depicted schematically in Figs. 1a and b.

The hadronic light-by-light (HLbL) contribution and the recent progress on its short-distance part [3] is discussed in Sect. 2. The main conclusion is that the quark-loop as often used in this respect really is the first term in a proper short-distance expansion. The lowest-order hadronic vacuum-polarization (HVP) and our estimate of electromagnetic finite volume contributions [4] is discussed in Sect. 3. The main conclusion here is that these corrections start at order $1 / L^{3}$ and not at order $1 / L^{2}$. This is a general property and we check it explicitly in the case of scalar QED.

\section{Hadronic light-by-light}

This is the contribution depicted in Fig. 1b. The problem here is this contribution mixes low- and high-energy contributions and thus as a consequence double-counting between hadronexchanges versus quark-gluon parts is an important aspect to consider. The hadronic object needed is the vector four-point function depicted in Fig. 1c. In general this has 138 Lorentz-structures but in four dimensions there are 41 independent combinations. Of these 12 combinations are needed in the limit $q_{4} \rightarrow 0$. A full analysis and references to earlier work can be found in [5]. Based on the dispersive method of [5] the long-distance contribution can clearly be brought under control. The major remaining part is now the intermediate and short-distance behaviour. The quark-loop has been used in this context before [6,7]. The operator product expansion (OPE) has been used in the HLbL context in many ways before, mainly to put restrictions on the behaviour of form-factors of hadrons but also to put constraints on the full vector four-point function in the kinematic limit $Q_{1}^{2} \approx Q_{2}^{2} \gg Q_{3}^{2}$ with $q_{i}^{2}=-Q_{i}^{2}$ and all momenta Euclidean [8]. 


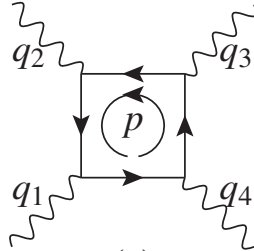

(a)

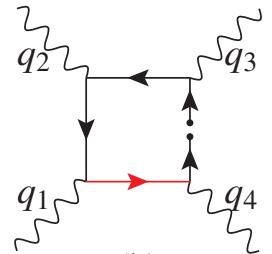

(b)

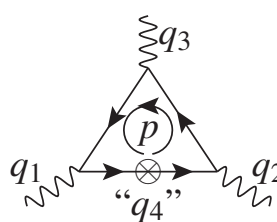

(c)

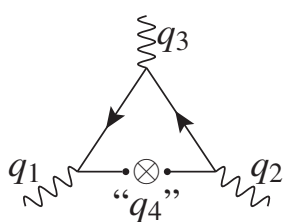

(d)

Figure 2: The quark-loop contribution: (a) the standard one, (b) a divergent OPE correction, (c) the quarkloop in the OPE in a background field, (d) the next order in the background field OPE with an insertion of the induced condensate.

\begin{tabular}{cccc}
\hline$Q_{\min }$ & quark-loop & $m_{u} X_{u}+m_{d} X_{d}$ & $m_{s} X_{s}$ \\
\hline $1 \mathrm{GeV}$ & $17.3 \times 10^{-11}$ & $5.40 \times 10^{-13}$ & $8.29 \times 10^{-13}$ \\
$2 \mathrm{GeV}$ & $4.35 \times 10^{-11}$ & $3.40 \times 10^{-14}$ & $5.22 \times 10^{-14}$ \\
\hline
\end{tabular}

Table 1: Numerical results for the quark-loop and the next term in the OPE.

We look now at the case where $Q_{1}^{2}, Q_{2}^{2}, Q_{3}^{2} \gg \Lambda_{Q C D}^{2}$. The quark-loop contribution depicted in Fig. $2 \mathrm{a}$ is finite in this limit but if we try to calculate higher order terms in the OPE, e.g. the $m_{q}\langle\bar{q} q\rangle$ contribution we get divergences when putting $q_{4} \rightarrow 0$. This is clearly seen in Fig. $2 \mathrm{~b}$ where the red propagator diverges in this limit. A similar problem occurred in QCD sum rules for the baryon magnetic moments [9] and we have adapted their method to the case at hand. We use an OPE in the presence of the background field and use the radial gauge for the background field. The latter allows to immediately take the limit $q_{4} \rightarrow 0$ and the lowest order contribution is depicted in Fig. 2c. The propagator with the crossed circle is the quark propagator in the background field. This contribution is exactly the same as the usual quark-loop calculated via Fig. 2a [3]. The next term is proportional to the induced condensate $\left\langle\bar{q} \sigma_{\alpha \beta} q\right\rangle \equiv e_{q} F_{\alpha \beta} X_{q}$. The quantities $X_{q}$ have been determined in lattice QCD and are about $40 \mathrm{MeV}$ [10]. The contribution to HLbL can be written using six functions [5] and for the next term these are given by ${ }^{1}$

$$
\begin{array}{rlrl}
\hat{\Pi}_{1} & =m_{q} X_{q} e_{q}^{4} \frac{-4\left(Q_{1}^{2}+Q_{2}^{2}-Q_{3}^{2}\right)}{Q_{1}^{2} Q_{2}^{2} Q_{3}^{4}}, & & \hat{\Pi}_{7}=0, \\
\hat{\Pi}_{4}=m_{q} X_{q} e_{q}^{4} \frac{8}{Q_{1}^{2} Q_{2}^{2} Q_{3}^{2}}, & \hat{\Pi}_{17}=m_{q} X_{q} e_{q}^{4} \frac{8}{Q_{1}^{2} Q_{2}^{2} Q_{3}^{4}}, \\
\hat{\Pi}_{54}=m_{q} X_{q} e_{q}^{4} \frac{-4\left(Q_{1}^{2}-Q_{2}^{2}\right)}{Q_{1}^{4} Q_{2}^{4} Q_{3}^{2}}, & \hat{\Pi}_{39}=0 .
\end{array}
$$

Note that they are suppressed w.r.t. the pure quark-loop by two powers of the hard scales, not four as would be expected from a first contribution arising from $\left\langle\alpha_{S} G^{2}\right\rangle$ or $m_{q}\langle\bar{q} q\rangle$.

Numerical results for the quark-loop and the next term in the OPE for $Q_{1}, Q_{2}, Q_{3} \geq Q_{\min }$, $m_{u}=m_{d}=m_{s}=0$ for the quark-loop and $m_{u}=m_{d}=5 \mathrm{MeV}$ and $m_{s}=100 \mathrm{MeV}$ for $m_{q} X_{q}$ are given in Tab. 1. To be noted that for the quark-loop the contribution above $1 \mathrm{GeV}$ is still $15 \%$ of

\footnotetext{
${ }^{1}$ These differ by a factor of -2 from the preliminary results shown at the conference.
} 
the total value of HLbL estimated. The quark-loop goes as $1 / Q_{\min }^{2}$ and the $m_{q} X_{q}$ contribution goes as $1 / Q_{\min }^{4}$. This can be shown using dimensional arguments. The second term is very small since both the values for $X_{q}$ and the relevant quark-masses are very small. Higher order terms will not have this suppression and are under investigation.

\section{Hadronic vacuum-polarization}

The lowest-order hadronic-vacuum-polarization contribution is the largest hadronic contribution to the muon anomalous magnetic moment. It can be determined via a dispersion relation directly from experiment and the precision of these determinations is about $0.5 \%$. Low-energy QCD can also be studied via lattice QCD. The present accuracy is a few \%, see e.g. [11], but the precision is expected to improve in the future. The precision needed requires that electromagnetic and other isospin breaking corrections are taken into account. The finite volume corrections are known to two-loop order in Chiral Perturbation Theory [12] but the electromagnetic finite volume corrections can be much larger since they are only suppressed by powers of the lattice size $L, 1 / L^{n}$ rather than exponentially, $\exp (-m L)$.

The main object here is the vector two-point function

$$
\Pi_{E M}^{\mu v}(q)=i \int d^{4} x e^{i q \cdot x}\langle 0| T\left(j_{a}^{\mu}(x) j_{b}^{v \dagger}(0)|0\rangle\right.
$$

with $j_{E M}^{\mu}=(2 / 3) j_{U}^{\mu}-(1 / 3) j_{D}^{\mu}-(1 / 3) j_{S}^{\mu}$ and $j_{Q}^{\mu}=\bar{q} \gamma^{\mu} q$. In the continuum we can write $\Pi_{E M}^{\mu v}(q)=$ $\left(q^{\mu} q^{v}-q^{2} g^{\mu v}\right) \Pi_{E M}\left(q^{2}\right)$ and the contribution to the anomalous magnetic moment is with a known positive weight function $v$ and $Q^{2}=-q^{2}$ :

$$
a_{\mu}=\int_{0}^{\infty} d Q^{2} v\left(Q^{2}\right)\left(-\Pi\left(Q^{2}\right)+\Pi(0)\right) .
$$

We first calculate the finite volume electromagnetic correction in scalar QED with Lagrangian

$$
\mathscr{L}=\left(\partial_{\mu} \Phi^{*}+i e A_{\mu} \Phi^{*}\right)\left(\partial_{\mu} \Phi-i e A_{\mu} \Phi\right)-m_{0}^{2} \Phi^{*} \Phi-\frac{1}{4} F_{\mu v} F^{\mu v} .
$$

The $\lambda\left(\Phi^{*} \Phi\right)^{2}$ is not needed to the order we are working. The photon corrections are calculated using the methods of [13] extended to two-loop order. The integral over photon momenta in loop integrals is replaced by the sum

$$
\int d^{d} k \frac{1}{k^{2}} \rightarrow \int d k^{0} \sum_{\vec{k}} \frac{1}{\left(k^{0}\right)^{2}-\vec{k}^{2}}
$$

and the sum needs regularizing. In $Q E D_{L}$ we do this by dropping the parts in the sum with $\vec{k}=0$. As an example take the two-loop integral

$$
S=\frac{1}{i^{2}} \int \frac{d^{d} l}{(2 \pi)^{d}} \frac{d^{d} k}{(2 \pi)^{d}} \frac{1}{k^{2}\left(l^{2}-m^{2}\right)\left((k+l-p)^{2}-m^{2}\right)} .
$$

The $l^{0}, k^{0}$ integrals are done via contour integration and we now write $\vec{k}=\frac{2 \pi}{L} \vec{n}$ and expand in $1 / L$. The $\vec{k}$ part can be written as

$$
\frac{1}{L^{d-1}} \sum_{\vec{n} \neq \overrightarrow{0}}=\int \frac{d^{d-1} k}{(2 \pi)^{d-1}}+\left[\frac{1}{L^{d-1}} \sum_{\vec{n} \neq \overrightarrow{0}}-\int \frac{d^{d-1} k}{(2 \pi)^{d-1}}\right]
$$




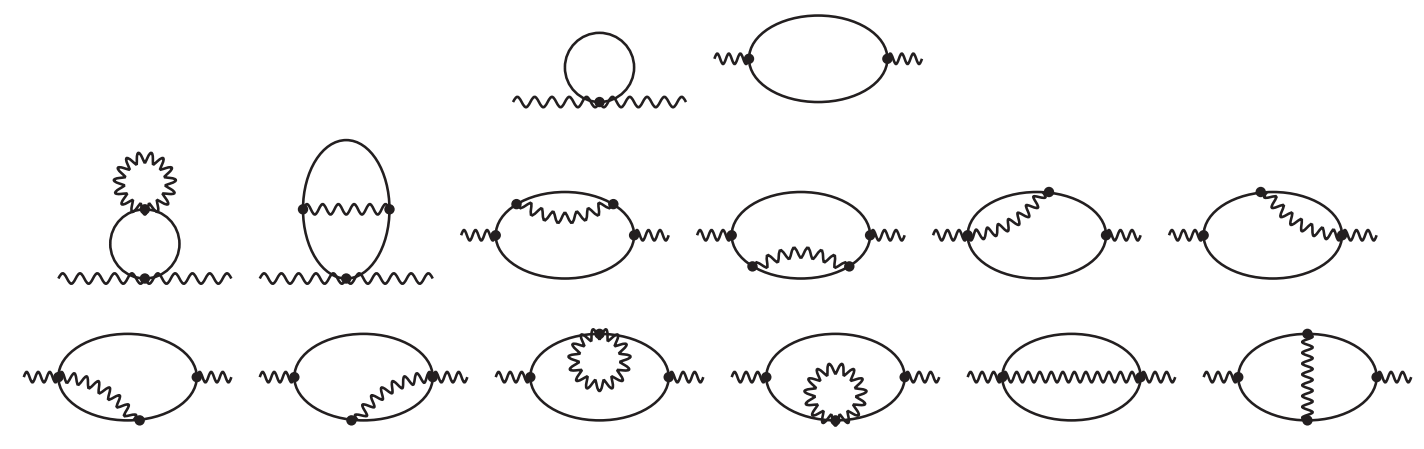

Figure 3: The diagrams to two-loop order of the vector two-point function in scalar QED. Lowest-order is the top line. Not shown are the diagrams involving counterterms and the disconnected contribution.

In the first term we resum the series in $1 / L$ which gives the infinite volume contribution. The quantity in brackets we define to be $\left(1 / L^{d-1}\right) \Delta_{\vec{n}}^{\prime}$ and the $1 / L^{n}$ dependence can be characterized via the coefficients $c_{m}=\Delta_{\vec{n}}^{\prime} \frac{1}{\vec{n}^{\mid m}}$. These are known numerically.

The correction to the mass is

$$
\frac{\Delta_{V} m^{2}}{m^{2}}=e^{2}\left(\frac{4 c_{2}}{16 \pi^{2} m L}+\frac{2 c_{1}}{16 \pi^{2} m^{2} L^{2}}+\mathscr{O}\left(\frac{1}{L^{4}}, e^{-m L}\right)\right)
$$

which agrees with the known result. Numerically this can be a very large correction. It starts at $1 / L$ since the photon and the pion can both be (almost) on-shell.

The two-point function can be calculated from the diagrams in Fig. 3 To be precise we calculated, with $t_{\mu \nu}$ the spatial part of $g_{\mu \nu}$

$$
\widetilde{\Pi}\left(\left(p^{0}\right)^{2}\right) \equiv \frac{-1}{3 p^{2}} t_{\mu v}\left(\Pi^{\mu v}(p)-\Pi^{\mu v}(p=0)\right)
$$

which reduces to $\Pi\left(p^{2}\right)$ in the infinite volume limit. In terms of the functions

$$
\Omega_{i j}\left(p^{2} / m^{2}\right)=\int \frac{d^{d-1} l}{(2 \pi)^{d-1}} \frac{m^{i+2 j-d+1}}{\left(\vec{l}^{2}+m^{2}\right)^{i / 2}\left(4 \vec{l}^{2}+4 m^{2}-p^{2}\right)^{j}}
$$

we obtain

$$
\widetilde{\Pi}\left(p^{2}\right)=+\frac{c_{0}}{m^{3} L^{3}}\left(-\frac{16}{3} \Omega_{0,3}-\frac{5}{3} \Omega_{2,2}+\frac{40}{9} \Omega_{2,3}-\frac{3}{8} \Omega_{4,1}+\frac{7}{6} \Omega_{4,2}+\frac{8}{9} \Omega_{4,3}\right)+\mathscr{O}\left(\frac{1}{L^{4}}, e^{-m L}\right) .
$$

Note that it starts only at order $1 / L^{3}$. This is because the two-point function is a neutral object and far away the photon sees only the dipole effect, not a charge. In the Euclidean all meson lines are off-shell as well. We have checked that our analytical result agrees well with the same result obtained using lattice perturbation theory for scalar QED as well as with a numerical evaluation putting scalar QED on a lattice. Details can be found in [4]. 


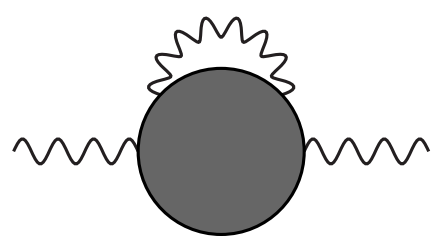

Figure 4: A generic QED correction to the vector two-point function.

The conclusion that the correction only starts at $1 / L^{3}$ is general. The correction can be seen as the generic diagram in Fig. 4. If we cut open the photon line it reduces the blob to the vector fourpoint function shown in Fig. 1c. That four-point function has no infra-red singularities, that can be seen from the analysis in [5] and references therein. Gauge-invariance enforces at least a factor cancelling the photon singular behaviour as discussed in more detail in [4]. The $1 / L^{3}$ behaviour is universal though the coefficient in front is not. For reasonable values of $L$ the finite volume correction is thus expected to be small and negligible for the precision needed in the near future.

\section{Conclusions}

This talk presented the main result of [3] and [4]. Both concern hadronic contributions to the muon anomalous magnetic moment. In the first we obtained that the usual quark-loop is indeed the first term in a systematic OPE. The next term was also calculated and found to be numerically small. In the second we showed that the electromagnetic finite volume corrections to the vector two-point function in general only start at order $1 / L^{3}$ and checked these results in scalar QED with a continuum method, lattice perturbation theory and a numerical lattice scalar QED calculation.

\section{Acknowledgements}

This work is supported in part by the Swedish Research Council grants contract numbers 201504089 and 2016-05996, and by the European Research Council (ERC) under the European Union's Horizon 2020 research and innovation programme under grant agreement No 668679.

\section{References}

[1] G. W. Bennett et al. [Muon g-2 Collaboration], "Final Report of the Muon E821 Anomalous Magnetic Moment Measurement at BNL," Phys. Rev. D 73 (2006) 072003 [hep-ex/0602035].

[2] F. Jegerlehner, "Muon $g-2$ theory: The hadronic part," EPJ Web Conf. 166 (2018) 00022 [arXiv:1705.00263 [hep-ph]].

[3] J. Bijnens, N. Hermansson-Truedsson and A. Rodríguez-Sánchez, "Short-distance constraints for the HLbL contribution to the muon anomalous magnetic moment," Phys. Lett. B 798C (2019) 134994 [arXiv:1908.03331 [hep-ph]].

[4] J. Bijnens, J. Harrison, N. Hermansson-Truedsson, T. Janowski, A. Jüttner and A. Portelli, "Electromagnetic finite-size effects to the hadronic vacuum polarization," Phys. Rev. D 100 (2019) no.1, 014508 [arXiv:1903.10591 [hep-lat]]. 
[5] G. Colangelo, M. Hoferichter, M. Procura and P. Stoffer, "Dispersion relation for hadronic light-by-light scattering: two-pion contributions,” JHEP 1704 (2017) 161 [arXiv:1702.07347 [hep-ph]].

[6] J. Bijnens, E. Pallante and J. Prades, "Analysis of the hadronic light by light contributions to the muon $g-2, ”$ Nucl. Phys. B 474 (1996) 379 [hep-ph/9511388].

[7] J. Bijnens and J. Prades, "The Hadronic Light-by-Light Contribution to the Muon Anomalous Magnetic Moment: Where do we stand?," Mod. Phys. Lett. A 22 (2007) 767 [hep-ph/0702170 [HEP-PH]].

[8] K. Melnikov and A. Vainshtein, "Hadronic light-by-light scattering contribution to the muon anomalous magnetic moment revisited," Phys. Rev. D 70 (2004) 113006 [hep-ph/0312226].

[9] B. L. Ioffe and A. V. Smilga, "Nucleon Magnetic Moments and Magnetic Properties of Vacuum in QCD,” Nucl. Phys. B 232 (1984) 109.

[10] G. S. Bali, F. Bruckmann, G. Endrodi, Z. Fodor, S. D. Katz and A. Schafer, "QCD quark condensate in external magnetic fields,” Phys. Rev. D 86 (2012) 071502 [arXiv:1206.4205 [hep-lat]].

[11] H. B. Meyer and H. Wittig, "Lattice QCD and the anomalous magnetic moment of the muon," Prog. Part. Nucl. Phys. 104 (2019) 46 [arXiv:1807.09370 [hep-lat]].

[12] J. Bijnens and J. Relefors, "Vector two-point functions in finite volume using partially quenched chiral perturbation theory at two loops," JHEP 1712 (2017) 114 [arXiv:1710.04479 [hep-lat]].

[13] Z. Davoudi, J. Harrison, A. Jüttner, A. Portelli and M. J. Savage, “Theoretical aspects of quantum electrodynamics in a finite volume with periodic boundary conditions," Phys. Rev. D 99 (2019) no.3, 034510 [arXiv:1810.05923 [hep-lat]]. 